\title{
Comparison of co-administration of amiodarone and rivaroxaban to co-administration of dronedarone and rivaroxaban for hemorrhage risks after atrial fibrillation ablation
}

\author{
Peng Zhang ${ }^{1}$ (1) $\cdot$ Maojing Wang ${ }^{1} \cdot$ Wenheng Liu $^{1} \cdot$ Pin Sun ${ }^{2} \cdot$ Shanglang Cai ${ }^{1}$ Yaqi Pan ${ }^{1} \cdot$ Qing Zhao $^{1}$
}

Received: 10 December 2021 / Accepted: 13 January 2022 / Published online: 20 January 2022

(c) The Author(s) 2022

\begin{abstract}
Purpose To investigate whether co-administration of antiarrhythmic dronedarone and anticoagulant rivaroxaban would increase the risks of hemorrhage after atrial fibrillation $(\mathrm{AF})$ ablation.

Methods A total of 100 patients with AF who underwent radiofrequency catheter ablation (CA) in the Department of Cardiology, the Affiliated Hospital of Qingdao University from 2019-12 to 2020-11 were included. Patients were divided into an oral dronedarone and rivaroxaban group ( $\mathrm{D}-\mathrm{R}$ group, $N=50)$ and an oral amiodarone and rivaroxaban group (A-R group, $N=50$ ) according to the postoperative antiarrhythmic and anticoagulation strategies. Patients in 2 groups were given propensity score matching (PSM) to obtain a sample with balanced inter-group covariates. A retrospective observational study was conducted. After 3 months of follow-up, the incidence of clinically relevant non-major bleeding (CRNMB), major hemorrhages, and early AF recurrence was observed.

Results After PSM, 41 patients were included in each group. With similarly distributed baseline characteristics and ablation characteristics after PSM, the CRNMB rate after AF ablation was significantly higher in the D-R group than in the A-R group (26.8\% versus $7.3 \%, P=0.02)$, and no major hemorrhages were detected in both groups. No significant difference was observed in the sinus rhythm maintenance rate between the D-R group and the A-R group (26.8\% vs. 22.0\%, $P=0.43)$. Conclusions Compared to co-administration of amiodarone and rivaroxaban, co-administration of dronedarone and rivaroxaban increases the risk of CRNMB but it does not increase the risk of major hemorrhages in blanking period after AF ablation.
\end{abstract}

Keywords Atrial fibrillation $\cdot$ Dronedarone $\cdot$ Amiodarone $\cdot$ Rivaroxaban $\cdot$ Hemorrhage

\section{Abbreviations}

AF Atrial fibrillation

DOACs Direct oral anticoagulants

ACT Activated whole blood coagulation time

CA Catheter ablation

PVI Pulmonary vein isolation

AI Ablation index

DCC Direct current synchronized cardioversion

CRNMB Clinically relevant non-major bleeding

BARC Bleeding Academic Research Consortium

Qing Zhao

zhaoqingqddx@163.com

1 Department of Cardiology, The Affiliated Hospital of Qingdao University, Qingdao 266000, Shandong, China

2 Department of Cardiac Ultrasound, The Affiliated Hospital of Qingdao University, Qingdao 266000, Shandong, China
ISTH International Society on Thrombosis and Haemostasis

PSM Propensity score matching

\section{Introduction}

Catheter ablation (CA) is a well-established treatment for the prevention of atrial fibrillation (AF) recurrences [1]. Dronedarone, an oral class III antiarrhythmic drug without iodine atoms in its structure, is often used to maintain sinus rhythm after CA and does not increase the incidence of thyroid toxicity [2]. However, dronedarone increases the plasma levels of direct oral anticoagulants (DOACs), such as rivaroxaban [3]. There are limited data on hemorrhage complications after co-administration of dronedarone and rivaroxaban. The current observational study evaluated whether co-administration of dronedarone and rivaroxaban would 
increase hemorrhage risks compared to co-administration of amiodarone and rivaroxaban after CA.

\section{Methods}

\subsection{Patients}

A total of 100 patients who had indication for CA and underwent CA for the first time in the Affiliated Hospital of Qingdao University from December 2019 to November 2020 were retrospectively reviewed. The patients were divided into an oral dronedarone and rivaroxaban group (D-R group, $N=50$ ), and an oral amiodarone and rivaroxaban group (A-R group, $N=50$ ) according to the postoperative antiarrhythmic and anticoagulation strategies.

The inclusion criteria were as follows: (1) patients with paroxysmal AF or persistent AF; (2) patients with nonvalvular AF. The exclusion criteria were as follows: (1) left atrial thrombosis detected by transesophageal echocardiography; (2) valvular AF (AF with moderate to severe mitral stenosis or mechanical valve replacement); (3) permanent AF; (4) warfarin use during the perioperative period; (5) major bleeding complications during surgery; (6) acute heart failure; (7) severe thyroid, lung, liver, and/or kidney dysfunction; and (8) age $<18$ or $>75$ years.

Patients in both groups were given propensity score matching (PSM) to obtain a sample with balanced intergroup covariates. The matching factors included age, AF types, left atrial diameter, left ventricular ejection fraction, cerebral embolism, hypertension, diabetes mellitus, $\mathrm{CHA}_{2} \mathrm{DS}_{2}$-VASc score, HAS-BLED score, preoperative medication, ablation characteristics, and procedural complications. According to the propensity score, the D-R group and the A-R group were matched at a ratio of 1:1. The matching tolerance was set to 0.02 .

\subsection{Radiofrequency catheter ablation methods}

All patients underwent transesophageal echocardiography within $48 \mathrm{~h}$ before ablation to exclude left atrial thrombosis. The right femoral vein was punctured, a 10-electrode coronary sinus catheter was placed in the coronary sinus, and then a Swartz $8.5 \mathrm{~F}$ sheath was inserted into the right atrium. The atrial septum puncture needle was guided along the sheath to the oval fossa to puncture the atrial septum. After a successful puncture, $100 \mathrm{IU} / \mathrm{kg}$ unfractionated heparin sodium was injected intravenously. The activated whole blood coagulation time (ACT) was monitored once every $0.5 \mathrm{~h}$ during the procedure. The dosage of unfractionated heparin sodium and protamine needed during the procedure was adjusted to stabilize the ACT value at $250 \sim 350 \mathrm{~s}$. The Swartz sheath was placed in the left atrium, and star mapping electrodes were placed along the sheath in the left atrium. The left atrium model was established under the guidance of the Carto system.

The ablation strategy of paroxysmal AF was pulmonary vein isolation (PVI). A $3.5-\mathrm{mm}$ ablation catheter (Smart Touch Johnson \& Johnson, New Brunswick, NJ, USA) was used to perform PVI in the bilateral pulmonary veins. The discharge energy in the anterior wall was $40 \mathrm{~W}$, the pump tube flow rate was $20 \mathrm{~mL} / \mathrm{min}$, and the limited ablation temperature was $43{ }^{\circ} \mathrm{C}$; consequently, the ablation was stopped when the ablation index (AI) reached $450 \sim 550$. Furthermore, when the discharge energy was $35 \mathrm{~W}$ at the top and posterior walls, the pump tube flow rate was $17 \mathrm{~mL} / \mathrm{min}$, and the limited ablation temperature was $43^{\circ} \mathrm{C}$; ablation was stopped when $\mathrm{AI}$ reached 350 400. Ablation was successful when both pulmonary veins reached bidirectional electrical isolation. Patients who did not convert to sinus rhythm successfully after PVI were subjected to direct current synchronized cardioversion (DCC).

The ablation strategy of persistent AF was PVI+left atrial apical line and mitral isthmus line ablation. The PVI ablation method was the same as for paroxysmal AF. When the parietal line of the left atrium was ablated and the ablation energy was $30 \mathrm{~W}$, the pump tube flow rate was $17 \mathrm{~mL} /$ min, the limited ablation temperature was $43{ }^{\circ} \mathrm{C}$, and the ablation was stopped when AI reached 400; when the ablation energy was $35 \mathrm{~W}$, the pump tube flow rate was $17 \mathrm{~mL} /$ min, the limited ablation temperature was $43{ }^{\circ} \mathrm{C}$, and the ablation was stopped when AI reached 400 450. Ablation was successful when both sides of the ablation line reached bidirectional electrical isolation, following which the patients with AF were given DCC after ablation.

\subsection{Perioperative antiarrhythmia and anticoagulation methods}

All patients were administered rivaroxaban $15 \mathrm{mg}$ orally for at least 1 month before $\mathrm{CA}$, which was stopped 1 day before the procedure, and the administration of antiarrhythmic drugs was stopped at five half-lives before the procedure. Under the condition of no bleeding complications, patients with a history of thyroid dysfunction, including hypothyroidism, subclinical hypothyroidism, and hyperthyroidism, were administered dronedarone and rivaroxaban orally $6 \mathrm{~h}$ after the procedure. For these patients, $400 \mathrm{mg}$ dronedarone was given twice a day, and $15 \mathrm{mg}$ rivaroxaban was given once a day. Patients with normal thyroid dysfunction were administered amiodarone and rivaroxaban orally $6 \mathrm{~h}$ after the procedure. The dosage of amiodarone was $200 \mathrm{mg}$ three times a day for the first 10 days and then it was adjusted to $200 \mathrm{mg}$ once a day after the first 10 days, and $15 \mathrm{mg}$ rivaroxaban was taken once a day. If the patient had bleeding complications during the operation, only dronedarone 
or amiodarone was administered after the procedure. The thromboembolism risks and bleeding risks were evaluated according to $\mathrm{CHA}_{2} \mathrm{DS}_{2}$-VAS score and HAS-BLED score. The maximum delay for oral anticoagulants was $48 \mathrm{~h}$ if the risks of bleeding outweigh the risks of thromboembolism especially in patients with HAS-BLED score $\geq 3$. All patients took antiarrhythmic drugs and anticoagulants orally for at least 3 months after CA.

\subsection{Postoperative follow-up}

All patients returned for follow-up at the outpatient clinic for 3 months after ablation. The main follow-up data collected included symptoms, signs, and tests of hemorrhage. Monthly body surface electrocardiogram and 24-h Holter monitoring were performed to record the recurrence of AF. Patients were instructed to immediately obtain a surface electrocardiogram at a local hospital if symptoms such as palpitation, chest tightness, or feelings of suffocation were suspected to be an $\mathrm{AF}$ recurrence.

\subsection{Data collection}

The observation indicators included as follows: (1) Clinically relevant non-major bleeding (CRNMB) was defined according to the criteria of Bleeding Academic Research Consortium (BARC) as any overt, actionable sign of hemorrhage that does not fit the criteria for types 3,4 , or 5 but meet at least one of the following criteria: (a) requiring nonsurgical, medical intervention by a healthcare professional, (b) leading to hospitalization or increased level of care, (c) prompting evaluation [4]. For example, petechia (subcutaneous bleeding $<2 \mathrm{~mm}$ in diameter), purpura (subcutaneous bleeding with a diameter of $3 \sim 5 \mathrm{~mm}$ ), ecchymosis (subcutaneous bleeding over $5 \mathrm{~mm}$ in diameter), small subcutaneous hematoma (hematoma diameter $\geq 3 \mathrm{~cm}$ measured by ultrasound), minor gastrointestinal hemorrhage (only positive by a fecal occult blood test, no melena or hematemesis), epistaxis, and other bleeding events that do not require blood transfusion or surgery and do not increase the number of hospitalization days; (2) Major bleeding was defined according to the criteria of International Society on Thrombosis and Haemostasis (ISTH) and BARC as clinically overt bleeding which was fatal or associated with any of the following: (a) a fall in hemoglobin level of $2 \mathrm{~g} / \mathrm{dL}$ or more or documented transfusion of at least 2 units of packed red blood cells, (b) involvement of a critical anatomical site (intracranial, spinal, ocular, pericardial, articular, intramuscular with compartment syndrome, retroperitoneal), (c) BARC types 3 to 5 were also considered a major bleeding [5]; (3) Early recurrence of AF: The first 3 months after the first ablation was a blank period during which any atrial arrhythmia (AF, atrial flutter, and atrial shock) occurrence was counted as early recurrence of AF. A strategy flow chart is illustrated in Fig. 1.

\subsection{Statistical analysis}

The statistical data of this study were processed using SPSS 19.0 and Prism 8.0 statistical software. The continuous variables are expressed as the mean \pm standard deviation and were compared using single-factor $t$-test statistics. Categorical variables are expressed as numbers and percentages and were processed using the $\chi^{2}$ test. Kaplan-Meier curves were used to display the cumulative bleeding complication-free survival and cumulative atrial arrhythmia-free survival after the first ablation. $P<0.05$ indicates a statistically significant difference.

\section{Results}

\subsection{Patient characteristics}

After PSM, 41 patients were included in each group. As shown in Table 1, the distributions of the patient baseline data did not show any statistically significant difference between the groups after PSM.

All patients successfully converted to sinus rhythm after CA. No cerebral embolism, pulmonary embolism, transient ischemic attack, cardiac tamponade, or major bleeding complications developed during the operation after PSM. There

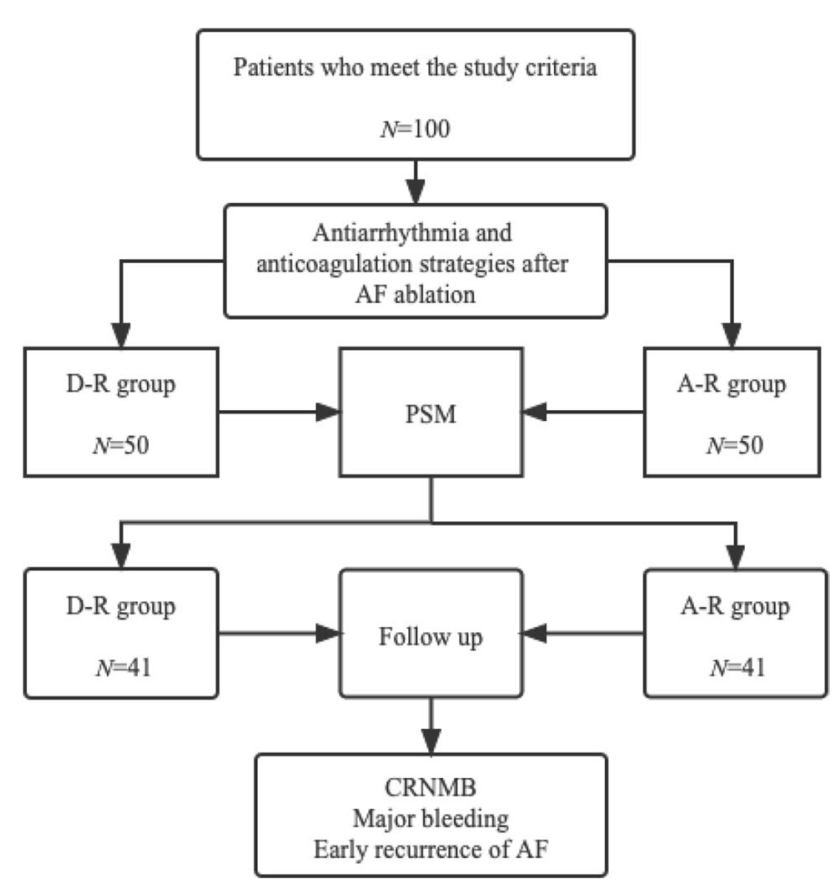

Fig. 1 Strategy flow chart for comparison of A-R group and D-R group. AF, atrial fibrillation; PSM, propensity score matching 
Table 1 Comparison of baseline clinical characteristics

\begin{tabular}{|c|c|c|c|c|c|c|}
\hline & \multicolumn{3}{|l|}{ Before PSM } & \multicolumn{3}{|l|}{ After PSM } \\
\hline & A-R group $(N=50)$ & D-R group $(N=50)$ & $P$ & A-R group $(N=41)$ & D-R group $(N=41)$ & $P$ \\
\hline Age (years) & $61.32 \pm 7.15$ & $62.92 \pm 8.10$ & 0.30 & $61.42 \pm 6.74$ & $61.93 \pm 8.11$ & 0.71 \\
\hline Men & $25(50.0 \%)$ & $22(44.0 \%)$ & 0.55 & $18(43.9 \%)$ & $18(43.9 \%)$ & - \\
\hline Paroxysmal atrial fibrillation & $29(58.0 \%)$ & $32(64.0 \%)$ & 0.54 & $30(73.2 \%)$ & $25(61.0 \%)$ & 0.24 \\
\hline Left atrial diameter $(\mathrm{mm})$ & $40.20 \pm 3.49$ & $40.02 \pm 3.40$ & 0.79 & $39.90 \pm 3.53$ & $39.95 \pm 3.60$ & 0.95 \\
\hline Left ventricular ejection fraction (\%) & $60.26 \pm 4.20$ & $59.92 \pm 3.13$ & 0.65 & $59.63 \pm 3.15$ & $59.76 \pm 2.36$ & 0.84 \\
\hline Cerebral embolism & $3(6.0 \%)$ & $4(8.0 \%)$ & 0.70 & $2(5.0 \%)$ & $2(5.0 \%)$ & - \\
\hline Hypertension & $24(48.0 \%)$ & $20(40.0 \%)$ & 0.42 & $20(48.8 \%)$ & $16(39.0 \%)$ & 0.37 \\
\hline Diabetes & $20(40.0 \%)$ & $15(30.0 \%)$ & 0.30 & $14(34.1 \%)$ & $13(31.7 \%)$ & 0.81 \\
\hline $\mathrm{CHA}_{2} \mathrm{DS}_{2}$-VASc score & $1.90 \pm 1.04$ & $2.06 \pm 1.20$ & 0.48 & $1.93 \pm 1.08$ & $1.88 \pm 1.10$ & 0.84 \\
\hline HAS-BLED score & $1.20 \pm 1.11$ & $1.18 \pm 0.98$ & 0.92 & $1.07 \pm 0.93$ & $1.15 \pm 1.04$ & 0.74 \\
\hline \multicolumn{7}{|l|}{ Preoperative medication } \\
\hline Propafenone & $6(12.0 \%)$ & $3(6.0 \%)$ & 0.30 & $6(14.6 \%)$ & $3(7.3 \%)$ & 0.29 \\
\hline Beta blocker & $13(26.0 \%)$ & $11(22.0 \%)$ & 0.64 & $12(29.3 \%)$ & $9(22.0 \%)$ & 0.45 \\
\hline Dronedarone & $4(8.0 \%)$ & $6(12.0 \%)$ & 0.51 & $2(5.0 \%)$ & $6(14.6 \%)$ & 0.14 \\
\hline Amiodarone & $7(14.0 \%)$ & $9(18.0 \%)$ & 0.59 & $6(14.6 \%)$ & $6(14.6 \%)$ & - \\
\hline Calcium channel blocker & $2(4.0 \%)$ & $5(10.0 \%)$ & 0.24 & $2(5.0 \%)$ & $4(9.8 \%)$ & 0.40 \\
\hline
\end{tabular}

Data are presented as mean + standard deviation or $n(\%)$

was no difference between D-R group and A-R group in minor bleeding complications, including small puncture site bleeding, hematoma, or ecchymosis (Table 2). All the patients who suffered from minor bleeding complications completely recovered within 2 days after surgery.

\subsection{Outcomes}

During 3-month of follow-up, D-R group had a significantly higher CRNMB rate than A-R group (26.8\% versus 7.3\%, $P=0.02$ ). Minor hemorrhage events occurred in 11 patients in D-R group, including petechia in 3 cases, purpura in 4 cases, and ecchymosis in 2 cases. Minor gastrointestinal hemorrhage occurred in 1 case, and epistaxis occurred in 1 case. Three cases of minor hemorrhage events were noted in A-R group, including petechia in 1 case, purpura in 2 cases, and a small subcutaneous hematoma in 1 case. However, the rates of petechia, purpura, ecchymosis, small subcutaneous hematoma, minor gastrointestinal hemorrhage, and epistaxis were numerically but not significantly different between the two groups. The minor hemorrhage-free survival rates of the two groups are shown in Fig. 2. No major bleeding

Table 2 Comparison of ablation characteristics and procedural complications

\begin{tabular}{|c|c|c|c|c|c|c|}
\hline & \multicolumn{3}{|l|}{ Before PSM } & \multicolumn{3}{|l|}{ After PSM } \\
\hline & A-R group $(N=50)$ & $\mathrm{D}-\mathrm{R}$ group $(N=50)$ & $P$ & A-R group $(N=41)$ & $\mathrm{D}-\mathrm{R}$ group $(N=41)$ & $P$ \\
\hline Operation time (min) & $122.76 \pm 18.02$ & $124.32 \pm 17.10$ & 0.71 & $122.49 \pm 14.82$ & $123.98 \pm 15.14$ & 0.65 \\
\hline Ablation time (min) & $35.44 \pm 5.16$ & $35.56 \pm 6.70$ & 0.94 & $34.83 \pm 4.31$ & $34.80 \pm 5.83$ & 0.98 \\
\hline X-ray exposure time (min) & $4.76 \pm 0.87$ & $4.59 \pm 0.71$ & 0.43 & $4.52 \pm 0.81$ & $4.29 \pm 0.77$ & 0.19 \\
\hline Direct current synchronized cardioversion & $12(24.0 \%)$ & $11(22.0 \%)$ & 0.81 & $10(24.4 \%)$ & $11(26.8 \%)$ & 0.80 \\
\hline $\begin{array}{l}\text { Success rate of instant pulmonary vein isola- } \\
\text { tion }\end{array}$ & $100 \%$ & $100 \%$ & - & $100 \%$ & $100 \%$ & - \\
\hline Cerebral embolism & 0 & 1 & 0.32 & 0 & 0 & - \\
\hline Pulmonary embolism & 0 & 0 & - & 0 & 0 & - \\
\hline Transient ischemic attack & 0 & 0 & - & 0 & 0 & - \\
\hline Cardiac tamponade & 0 & 0 & - & 0 & 0 & - \\
\hline Minor bleeding complications & $6(12.0 \%)$ & $8(16.0 \%)$ & 0.56 & $6(14.6 \%)$ & $6(14.6 \%)$ & - \\
\hline Major bleeding complications & 0 & 0 & - & 0 & 0 & - \\
\hline
\end{tabular}

Data are presented as mean + standard deviation or $n(\%)$ 


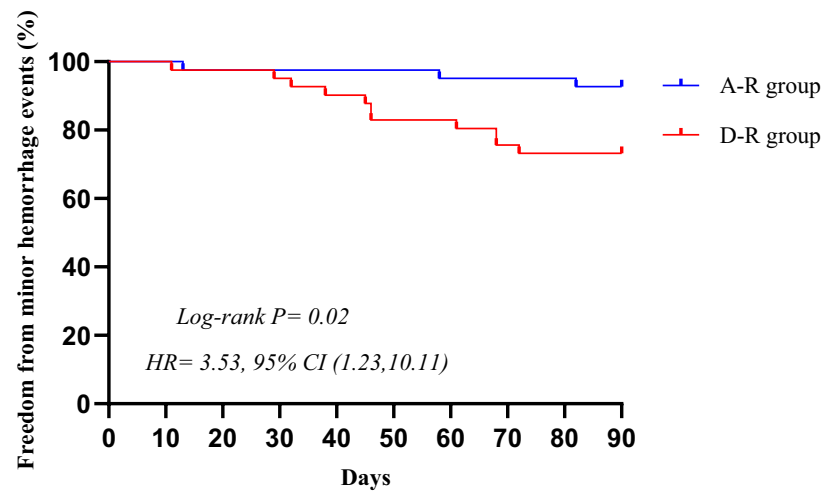

Fig. 2 Freedom from minor hemorrhage after AF ablation for D-R group and A-R group. At 3-month follow-up, there were differences between the two groups $(P=0.02)$. AF, atrial fibrillation

complications developed in either group during follow-up. Details of the hemorrhage events are presented in Table 3.

During 3-month of follow-up, the early AF recurrence rate was numerically but not significantly higher in the D-R group than in the A-R group (26.8\% vs. $22.0 \%, P=0.43$ ). The early AF recurrence-free survival rates of the two groups are shown in Fig. 3.

\section{Discussion}

The main findings of the present observational, single-center study are as follows: (1) Co-administration of dronedarone and rivaroxaban yielded a significantly higher risk of CRNMB for patients with paroxysmal or persistent AF but did not increase the risk of major hemorrhage after CA. (2) Dronedarone did not increase the early AF recurrence rate after the first ablation compared to amiodarone.

AF catheter ablation is effective in maintaining sinus rhythm in patients with paroxysmal and persistent AF [6, 7]. The first 3 months after the first ablation was a blanking period during which any atrial arrhythmia occurrence was not counted as an AF recurrence but was the strongest

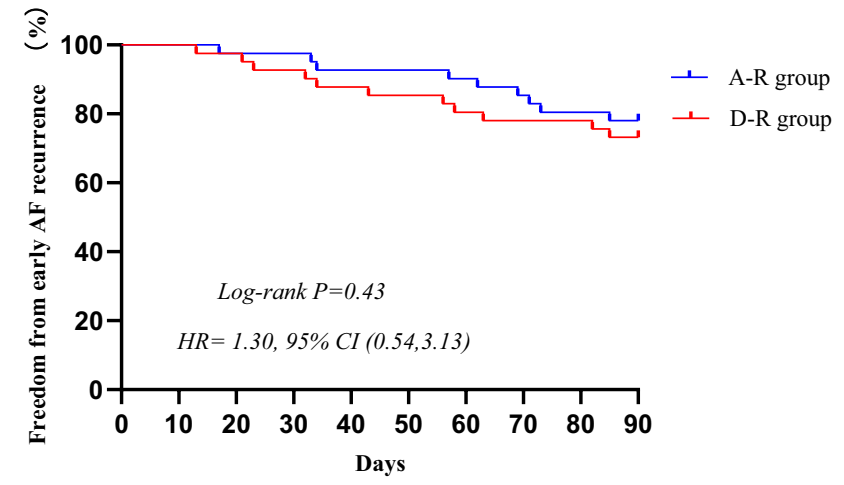

Fig. 3 Freedom from early AF recurrence after AF ablation for D-R group and A-R group. At 3-month follow-up, there were no differences between the two groups $(P=0.43)$. AF, atrial fibrillation

predictor for AF recurrence in the future [8]. Continuing antiarrhythmic drug treatment for 3 months after CA was recommended to reduce early AF recurrences during the blanking period [9]. Additionally, oral anticoagulant therapy is continued for at least 2 months following CA in all patients [10].

The plasma levels of DOACs can be influenced by various drug-drug interactions and consequently influence anticoagulant activity [11]. At present, the interactions between amiodarone and DOACs have been noted [12]. However, relevant data on co-administration of dronedarone and rivaroxaban are lacking. The present study demonstrated that co-administration of dronedarone and rivaroxaban increased the CRNMB risks, but it is still a relatively safe treatment option because it did not increase the incidence of major bleeding events in short-term applications. In a recent study of 23 patients with paroxysmal AF for an average 9.1-month follow-up, concomitant use of dronedarone and rivaroxaban was not associated with significant adverse events, including major bleeding [13]. A multicenter study found that co-administration of dronedarone and DOACs does not increase the risk of massive hemorrhage [14], which is consistent with our results. An
Table 3 Comparison of hemorrhage events during follow-up

\begin{tabular}{llll}
\hline & A-R group $(N=41)$ & D-R group $(N=41)$ & $P$ \\
\hline Minor hemorrhage events & $3(7.3 \%)$ & $11(26.8 \%)$ & 0.02 \\
Petechia & $1(2.4 \%)$ & $3(7.3 \%)$ & 0.31 \\
Purpura & $1(2.4 \%)$ & $4(9.8 \%)$ & 0.24 \\
Ecchymosis & 0 & $2(4.9 \%)$ & 0.56 \\
Small subcutaneous hematoma & $1(2.4 \%)$ & 0 & 0.32 \\
Minor gastrointestinal hemorrhage & 0 & $1(2.4 \%)$ & 0.32 \\
Epistaxis & 0 & $1(2.4 \%)$ & 0.32 \\
Major bleeding events & 0 & 0 & - \\
\hline
\end{tabular}

Data are presented as $n(\%)$ 
increase in the blood drug concentration of DOACs might not be related to an increased risk of massive hemorrhage.

Rivaroxaban is mainly resecreted into the gut via a P-glycoprotein transporter and metabolized by cytochrome P3A4 (CYP3A4) [15]. Dronedarone is an inhibitor of P-glycoprotein and CYP3A4 [12], which may result in higher plasma levels of rivaroxaban and lead to an increased risk of CRNMB. Regardless, co-administration of dronedarone and rivaroxaban can be used as a rhythm-controlling and anticoagulation strategy after CA, but the signs and tests of bleeding need to be monitored in real time.

Amiodarone is the most effective antiarrhythmic drug and shows lower AF recurrence than dronedarone [16], but numerous extracardiac side effects, such as thyroid toxicity, make it a second-line treatment [17]. Although less effective than amiodarone in rhythm control, dronedarone may be a preferable first choice with few extracardiac side effects [18]. The present study showed that dronedarone and amiodarone have comparable abilities to maintain sinus rhythm in the blanking period after CA. However, it is currently thought that dronedarone is less efficacious than amiodarone in maintaining sinus rhythm [19]. A possible explanation for the difference in results is the short follow-up time in this study; only the recurrence rate of atrial fibrillation in blanking period was evaluated. The recurrence of $\mathrm{AF}$ in blanking period cannot be used as an indicator to evaluate the effect of antiarrhythmic drugs.

The present study is limited by its single-center nature. A larger multicenter trial should be performed to elevate the hemorrhage risks of co-administration of dronedarone and rivaroxaban. Moreover, the judgment of AF recurrence mainly depends on symptoms and 24-h Holter monitoring, which does not rule out the possibility that patients with mild symptoms are not undergoing electrocardiogram examinations when $\mathrm{AF}$ recurs.

\section{Conclusions}

Compared to co-administration of amiodarone and rivaroxaban, co-administration of dronedarone and rivaroxaban increases the risk of CRNMB but it does not increase the risk of major hemorrhage in blanking period after CA.

Acknowledgements The authors thank Qingdao University for the assistance with statistical analysis.

\footnotetext{
Author contribution All authors contributed to the study conception and design. Mao-Jing Wang contributed to conceptualization; Qing Zhao contributed to formal analysis; Peng Zhang was responsible for the data curation and original draft preparation; Pin Sun, Ya-Qi Pan, and Wen-Heng Liu contributed to review and editing; and Shang-Lang Cai contributed to supervision. All authors have approved the final version for submission.
}

\section{Declarations}

Data access statement All data are provided in full in the results section of this paper.

This study complied with the 1975 Helsinki Ethics Guide and was approved by the Ethics Committee of The Affiliated Hospital of Qingdao University (No. QYFYWZLL 26013). Informed consent was obtained from the patients and their families.

Conflict of interest The authors declare no competing interests.

Open Access This article is licensed under a Creative Commons Attribution 4.0 International License, which permits use, sharing, adaptation, distribution and reproduction in any medium or format, as long as you give appropriate credit to the original author(s) and the source, provide a link to the Creative Commons licence, and indicate if changes were made. The images or other third party material in this article are included in the article's Creative Commons licence, unless indicated otherwise in a credit line to the material. If material is not included in the article's Creative Commons licence and your intended use is not permitted by statutory regulation or exceeds the permitted use, you will need to obtain permission directly from the copyright holder. To view a copy of this licence, visit http://creativecommons.org/licenses/by/4.0/.

\section{References}

1. Arbelo E, Brugada J, Blomström-Lundqvist C. Contemporary management of patients undergoing atrial fibrillation ablation: in-hospital and 1-year follow-up findings from the ESC-EHRA atrial fibrillation ablation long-term registry[J]. Eur Heart J. 2017;38(17):1303-16. https://doi.org/10.1093/eurheartj/ehw564.

2. Patel MR, Mahaffey KW, Garg J. Rivaroxaban versus warfarin in nonvalvular atrial fibrillation[J]. N Engl J Med. 2011;365(10):883-891. https://doi.org/10.1056/NEJMoa1009638.

3. Kirchhof P, Benussi S, Kotecha D. 2016 ESC guidelines for the management of atrial fibrillation developed in collaboration with EACTS[J]. Kardiologia Polska (Polish Heart Journal). 2016;74(12):1359-469. https://doi.org/10.5603/KP.2016.0172.

4. Kaatz S, Ahmad D, Spyropoulos AC, et al. Definition of clinically relevant non-major bleeding in studies of anticoagulants in atrial fibrillation and venous thromboembolic disease in non-surgical patients: communication from the SSC of the ISTH[J]. J Thromb Haemost. 2015;13(11):2119-26. https://doi.org/10.1111/jth. 13140.

5. Franco L, Becattini C, Beyer-Westendorf J, et al. Definition of major bleeding: prognostic classification[J]. J Thromb Haemost. 2020;18(11):2852-60. https://doi.org/10.1111/jth.15048.

6. Kuck KH, Brugada J, Fürnkranz A, et al. Cryoballoon or radiofrequency ablation for paroxysmal atrial fibrillation[J]. N Engl J Med. 2016;374(23):2235-45. https://doi.org/10.1056/NEJMo a1602014.

7. Chen C, Zhou X, Zhu M, et al. Catheter ablation versus medical therapy for patients with persistent atrial fibrillation: a systematic review and meta-analysis of evidence from randomized controlled trials[J]. J Interv Card Electrophysiol. 2018;52(1):9-18. https:// doi.org/10.1007/s10840-018-0349-8.

8. Kornej J, Schumacher K, Dinov B. Prediction of electro-anatomical substrate and arrhythmia recurrences using APPLE, DRFLASH and MB-LATER scores in patients with atrial fibrillation undergoing catheter ablation[J]. Sci Rep. 2018;8(1):1-7. https:// doi.org/10.1038/s41598-018-31133-x. 
9. Kaitani K, Inoue K, Kobori A. Efficacy of antiarrhythmic drugs short-term use after catheter ablation for atrial fibrillation (EASTAF) trial[J]. Eur Heart J. 2016;37(7):610-8. https://doi.org/10. 1093/eurheartj/ehv501.

10. Mardigyan V, Verma A, Birnie D. Anticoagulation management pre-and post atrial fibrillation ablation: a survey of Canadian centres[J]. Can J Cardiol. 2013;29(2):219-23. https://doi.org/10. 1016/j.cjca.2012.04.013.

11. Fitzgerald JL, Howes LG. Drug interactions of direct-acting oral anticoagulants[J]. Drug Saf. 2016;39(9):841-5. https://doi.org/10. 1007/s40264-016-0443-8.

12. Chiang CE, Okumura K, Zhang S. 2017 consensus of the Asia Pacific Heart Rhythm Society on stroke prevention in atrial fibrillation[J]. J Arrhythm. 2017;33(4):345-67. https://doi.org/ 10.1016/j.joa.2017.05.004.

13. Escobar C, Arceluz M, Montes de Oca R. Concomitant rivaroxaban and dronedarone administration in patients with nonvalvular atrial fibrillation[J]. Revista espanola de cardiologia (English ed.), 2016, 70(2): 121-122. https://doi.org/10.1016/j.rec.2016.06.018.

14. Chang SH, Chou IJ, Yeh YH. Association between use of nonvitamin $\mathrm{K}$ oral anticoagulants with and without concurrent medications and risk of major bleeding in nonvalvular atrial fibrillation[J]. JAMA. 2017;318(13):1250-9. https://doi.org/10. 1001/jama.2017.13883.
15. Mueck W, Kubitza D, Becka M. Co-administration of rivaroxaban with drugs that share its elimination pathways: pharmacokinetic effects in healthy subjects[J]. Br J Clin Pharmacol. 2013;76(3):455-66. https://doi.org/10.1111/bcp.12075.

16. Lafuente-Lafuente C, Valembois L, Bergmann J F. Antiarrhythmics for maintaining sinus rhythm after cardioversion of atrial fibrillation[J]. Cochrane Database of Systematic Reviews, 2015 (3); DOI: https://doi.org/10.1002/14651858.CD005049.pub4.

17. Epstein AE, Olshansky B, Naccarelli GV. Practical management guide for clinicians who treat patients with amiodarone $[\mathrm{J}]$. Am J Med. 2016;129(5):468-75. https://doi.org/10.1016/j.amjmed. 2015.08.039.

18. Wu C, Tcherny-Lessenot S, Dai W, et al. Assessing the risk for peripheral neuropathy in patients treated with dronedarone compared with that in other antiarrhythmics[J]. Clinical therapeutics, 2018, 40(3): 450-455. e1; https://doi.org/10.1016/j.clinthera. 2018.01.015.

19. Piccini JP, Hasselblad V, Peterson ED. Comparative efficacy of dronedarone and amiodarone for the maintenance of sinus rhythm in patients with atrial fibrillation[J]. J Am Coll Cardiol. 2009;54(12):1089-95. https://doi.org/10.1016/j.jacc.2009.04.085.

Publisher's note Springer Nature remains neutral with regard to jurisdictional claims in published maps and institutional affiliations. 